\title{
Type 1 Astrocytes Inhibit Myelination by Adult Rat Oligodendrocytes in vitro
}

\author{
Charles L. Rosen, ${ }^{a}$ Richard P. Bunge, March D. Ard, ${ }^{b}$ and Patrick M. Wood \\ Miami Project, University of Miami School of Medicine, Miami, Florida 33136
}

\begin{abstract}
We have determined the effect of Type 1 astrocytes on the myelination of dorsal root ganglion cell axons by oligodendrocytes obtained from adult animals. Experiments were initiated by the addition of oligodendrocytes [purified either by density gradient centrifugation and treatment in culture with 5-fluorodeoxyuridine (FdU) or by fluorescence-activated cell sorting after immunostaining with antigalactocerebroside antibody] to cultures of purified neurons. In control conditions, the added oligodendrocytes proliferate and, after 4 weeks, accomplish substantial myelination of the sensory axons. Type 1 astrocytes (purified from cultures of dissociated newborn rat cerebral cortex by vigorous shaking to remove less adherent cells) or fibroblasts (purified from cultures of cranial periosteum by serial replating) were added to some of these cultures after the oligodendrocytes had attached and started to proliferate. We observed that the added Type 1 astrocytes, but not the added fibroblasts, strongly inhibited myelination and caused decreased oligodendrocyte proliferation or survival. These effects of added Type 1 astrocytes were reproduced with Type 1 astrocyteconditioned medium. We conclude that Type 1 astrocytes can release soluble factors that inhibit oligodendrocyte myelination.
\end{abstract}

The physical proximity of astrocytes and oligodendrocytes in all regions of the CNS and their invariant sequential appearance (first astrocytes and then oligodendrocytes) in development suggest that astrocytes may influence oligodendrocyte function. Although the relationship between astrocytes and oligodendrocytes undoubtedly changes as the tissue develops and then matures, it is generally accepted that the role of the astrocyte is supportive of oligodendrocyte function. When this normal relationship is disturbed, as in injury or in demyelination, a prominent astrocyte response often occurs. In some situations, this response can produce astroglial scars, as in chronic lesions in

\footnotetext{
Received Sept. 29, 1988; revised Mar. 15, 1989; accepted Mar. 23, 1989.

We thank Dr. Junming Le for performing the assays for tumor nccrosis factor, Mr. Alon Mogilner for assistance in computer programming, and Drs. L. Eng, B. Ranscht and M. Schachner for gifts of antibodies. In addition, we would like to thank Ms. Susan Mantia for preparation of the manuscript and Mrs. Artree James for technical assistance. This work was supported by grant RG1118 from the National Multiple Sclerosis Society. M.D.A. was supported by a fellowship from the National Multiple Sclerosis Society.

Correspondence should be addressed to Dr. Patrick Wood, Miami Project University of Miami School of Medicine, 1600 N.W. 10th Avenue, R-48, Miami, FL 33136.

Present address: Department of Cell Biology, New York University School of Medicine, 550 1st Avenue, New York, NY 10016.

b Present address: Department of Anatomy, University of Mississippi Medical Center, 2500 N. State Street, Jackson, MS 39216-4505.
}

Copyright @ 1989 Society for Neuroscience 0270-6474/89/103371-09\$02.00/0 patients with multiple sclerosis. Whether this astroglial response promotes or inhibits the recovery of normal oligodendrocyte function is not clear. It has been observed (Blakemore, 1978) in an animal model of chemically induced demyelination-remyelination that remyelination does not occur when astrocyte numbers are low and when the myelin debris is not completely removed. Since astrocytes are thought to participate in the phagocytosis of myelin debris during demyelination, these observations suggest that a weak astrocyte response might restrict remyelination. On the other hand, there is a strong correlation between severe astrogliosis and the failure of effective remyelination (Ludwin, 1981; Prineas, 1985), implying that in some situations, astrocytes might have negative effects on oligodendrocyte function.

Our view of astrocyte function has been expanded considerably by recent demonstrations of two phenotypically distinct forms (Raff et al., 1983). Type 1 astrocytes appear early in development and are generated in large numbers when CNS tissue from embryonic or newborn animals is dissociated and cultured (Raff et al., 1984). They often display a flattened, epithelial morphology in culture. Type 2 astrocytes are defined by the expression on their surfaces of the antigen recognized by the monoclonal antibody A2B5. They are believed to derive from multipotential cells, which also express the A2B5 antigen and which may also develop into oligodendrocytes. Type 2 astrocytes are generated after oligodendrocytes in cultures of early postnatal optic nerve and are generated with a shorter delay in cultures of optic nerve from older animals (Raff et al., 1983). These astrocytes display a stellate morphology in culture. It has been suggested that they are also generated subsequent to oligodendrocytes in vivo, that they proliferate to eventually outnumber the Type 1 astrocytes, and that they may play some role in nodal physiology (Miller et al., 1985; ffrench-Constant and Raff, 1986). However, this view conflicts with earlier studies (Skoff et al., 1976) that demonstrated that most of the astrocytes in the mature rat optic nerve are generated before oligodendrocytes and should, therefore, be of the Type 1 phenotype. Thus, the relative abundance of Type 1 and Type 2 astrocytes in vivo remains controversial. In recent tissue culture experiments, Type 1 astrocytes have been shown to secrete a factor that promotes the division of oligodendrocyte-Type 2 astrocyte progenitors and regulates the timing of their differentiation into oligodendrocytes (Noble et al., 1988; Raff et al., 1988; Richardson et al., 1988). This factor has been shown to be similar or identical to platelet-derived growth factor. There is some evidence that the production of platelet-derived growth factor in the CNS parallels the development of astrocytes, suggesting that this platelet-derived growth factor-mediated interaction may play a role in gliogenesis in vivo; i.e., as the number of astrocytes increases, 
more platelet-derived growth factor may become available to stimulate division of oligodendrocyte precursors (Richardson et al., 1988). These results indicate that Type 1 astrocytes may exert a direct positive influence on normal oligodendrocyte development in vivo. There is some evidence that the astrocytes that proliferate in reaction to injury or demyelination also belong to the type 1 family (Miller et al., 1986). Thus, it might be expected, on the basis of the tissue culture experiments cited, that the proliferation of Type 1 astrocytes in vivo would promote oligodendrocyte proliferation and remyelination.

In this report, we describe tissue culture experiments designed to ascertain the effect of Type 1 astrocytes on the myelination of sensory axons by oligodendrocytes obtained from adult animals. In these experiments, the oligodendrocytes, purified either by density gradient centrifugation and antimitotic treatment or by fluorescence-activated cell sorting, were added to cultures of purified neurons to initiate myelination. Type 1 astrocytes, purified from cultures of dissociated newborn rat cerebral cortex by the selective adhesion method of McCarthy and de Vellis (1980) as modified by Noble et al. (1984), were added to these cultures after the oligodendrocytes had attached and started to proliferate. It was observed under these conditions that both astrocytes and astrocyte-conditioned medium strongly blocked myelin formation. Preliminary observations indicated that astrocytes also inhibited oligodendrocyte proliferation. Thus, soluble factors released by Type 1 astrocytes could be shown to be inhibitory to oligodendrocyte function in a culture system normally allowing rapid proliferation and accumulation of oligodendrocytes and extensive CNS myelin formation.

\section{Materials and Methods}

Neuronal cultures. The method of culturing cervical dorsal root ganglion neurons obtained from embryonic day 15 rat was described previously (Wood and Williams, 1984). Briefly, ganglia were removed from the embryo, treated with trypsin, and mechanically dissociated. The dissociated cells from 2 ganglia were plated in 1 drop of Medium 1 [Eagle's minimum essential medium (MEM) supplemented with heat-inactivated human placental serum (HIHPS) to $10 \%$, glucose to $0.4 \%$ (wt/ vol), and crude nerve growth factor (50 biological units $/ \mathrm{ml})$ ] in the center of $25 \mathrm{~mm}$ Aclar dishes coated with ammoniated collagen (Bornstein, 1958). The cultures were subsequently treated with the antimitotic agent 5-fluorodeoxyuridine (FdU) to remove fibroblasts and Schwann cells and maintained on Medium 1 until the oligodendrocytes were added.

Oligodendrocyte cultures. Cultures enriched with oligodendrocytes were obtained from the spinal cord of 3-month-old female rats. A laminectomy was performed, and the cord was dissected out and placed in Leibovitz medium (L-15, Gibco) at $4^{\circ} \mathrm{C}$. All meningeal tissues were removed, and the cord was cut sagittally into halves, minced into cubes (approximately $0.50 \mathrm{~mm}^{3}$ ), and incubated in $0.25 \%$ trypsin (United States Biochemical, $3 \times$ crystallized) and $40 \mu \mathrm{g} / \mathrm{ml}$ DNAase I, Type 2 (Sigma) in Earle's Balanced Salt Solution (EBSS) for $1 \mathrm{hr}$ in $5 \% \mathrm{CO}_{2}$ on a rotary shaker at $37^{\circ} \mathrm{C}$. Following incubation, enzyme activity was inhibited by adding HIHPS. Cells and tissue fragments were collected by centrifugation, resuspended in L-15 $+10 \%$ HIHPS (LS Medium) and triturated through a pipette with a tip diameter of about $0.5 \mathrm{~mm}$. The cell suspension was diluted with LS medium and filtered through a $15-\mu \mathrm{m}$ (pore size) Nitex filter. The filtrate $(\sim 5 \mathrm{ml})$ was added to 3.0 $\mathrm{ml}$ of $80 \%$ Percoll in $0.25 \mathrm{M}$ sucrose with $0.02 \mathrm{M}$ phosphate buffer. To this was added $1.5 \mathrm{ml} \mathrm{LS}$ medium to bring the final volume to $9.5 \mathrm{ml}$. The cell-Percoll suspension was thoroughly mixed and centrifuged at $30,000 \times g$ for $45 \mathrm{~min}$ at $4^{\circ} \mathrm{C}$. Myelin and cell debris were discarded, and the wide band of cells immediately above the red blood cell band was collected. These cells were rinsed in LS medium, centrifuged at 400 $\times g$ for $10 \mathrm{~min}$, and resuspended in $\sim 1.0 \mathrm{ml}$ of Medium 1 . The cells were filtered through a Nitex filter with a pore size of $15 \mu \mathrm{m}$ and plated onto a $60-\mathrm{mm}$ plastic dish coated with air-dried rat tail collagen (Bornstein, 1958; Wood, 1976). To rid cultures of dividing astrocytes while preserving nondividing oligodendrocytes, cultures were exposed to $\mathrm{Me}$ - dium 1 with $10^{5} \mathrm{FdU}$ on $\mathrm{d} \mathrm{2-4,6-8}$, and 11-13. Since axons are mitogenic for oligodendrocytes (Wood and Bunge, 1986a), the FdUcontaining medium was rinsed away before adding oligodendrocytes to dorsal root ganglion neurons. Cell suspensions prepared from these cultures for replating onto neuronal cultures were $>95 \%$ GalC-positive oligodendrocytes and $<2.5 \%$ GFAP-positive astrocytes.

Oligodendrocytes were also purified by fluorescence-activated cell sorting. A cell suspension was prepared from 3-month-old spinal cord as described above through the Percoll separation step. The cells (from one rat) were collected by centrifugation and resuspended in $0.5 \mathrm{ml}$ of hybridoma supernatant containing mouse monoclonal anti-GalC antibody (gift of Dr. Barbara Ranscht). (The supernatant was generated by incubating confluent hybridoma cultures in Dulbecco's Modified Eagle's Medium, buffered with $25 \mathrm{~mm}$ Hepes buffer and containing $5 \%$ heatinactivated horse serum, for $48 \mathrm{hr}$ at $37^{\circ} \mathrm{C}$.) The cells were exposed to the supernatant for $30 \mathrm{~min}$ at room temperature; during this period they were resuspended at 5 -min intervals. Fifty microliters of affinity purified fluorescein-conjugated goat antimouse IgG (Cappel, diluted 1:5) were added to the cell suspension, and the incubation was carried out for an additional $30 \mathrm{~min}$ with gentle trituration at 5 -min intervals. The suspension was then diluted to $3.0 \mathrm{ml}$ with LS medium and filtered through $15-\mu \mathrm{m}$ Nitex. This suspension was subjected to sorting on a Becton-Dickinson 440 cell sorter equipped with a 5 -W argon laser. The sorted cells were approximately $90-95 \% \mathrm{GalC}+$ oligodendrocytes as confirmed by cell counting in a hemocytometer. These cells were resuspended in Medium 1 and plated directly onto neuronal cultures. Cultures stained $24 \mathrm{hr}$ after the addition of those purified oligodendrocytes were completely devoid of GFAP+ astrocytes.

Astrocyte cultures. Astrocytes were obtained from the cerebral cortex of 1-d-old rats as described by McCarthy and de Vellis (1980) and as modified by Noble et al. (1984). Briefly, after dissection and enzymatic digestion, the tissue was dissociated to produce a single cell suspension, which was plated in Dulbecco's Modified Eagle's Medium with $10 \%$ fetal bovine serum in T-flasks. After 7-14 d in culture, a confluent monolayer of flat, firmly adhering cells covered the bottoms of the flasks, while loosely adherent cells were present on top of the monolayer. The loosely adherent cells were shaken off to yield cultures that were predominantly astrocytes. These cultures were subsequently treated with two 48-hr pulses of FdU $\left(10^{-5} \mathrm{M}\right)$ in the same medium. The composition of purified cultures was determined by immunostaining for glial fibrillary acidic protein (GFAP), an astrocyte marker (Bignami et al., 1972), anti-thy 1.1, a fibroblast marker (Raff et al., 1979), and A2B5, a Type 2 astrocyte-specific marker (Raff el al., 1983; Raff et al., 1984). AntiGFAP was a gift of Dr. Larry Eng. Hybridoma cell lines producing the other antibodies were purchased from American Type Culture Collection. Astrocytes (GFAP+ cells) accounted for $95 \%$ of the cells in these cultures. Of these, $94 \%$ were Type 1 (A2B5-) astrocytes, and only $1 \%$ were Type 2 (A2B5+) astrocytes. Three percent of the cells stained positive for anti-thy 1.1 .

Fibroblast cultures. Fragments of cranial periosteum were dissected from neonatal rats and grown for 7-10 d on a substrate of ammoniated collagen in MEM containing 10\% fetal bovine serum (Hyclone). Remnants of the periosteal explants were excised, and the cells remaining in the outgrowth area, which appeared to be morphologically homogeneous, were suspended by sequential incubations in $0.05 \%$ collagenase (Worthington, CLSPA grade) in EBSS and in $0.25 \%$ trypsin in $\mathrm{Ca}^{2+}$, $\mathrm{Mg}^{2+}$-free Hank's Balanced Salt Solution (CMFHBSS), each for $30 \mathrm{~min}$ at $35^{\circ} \mathrm{C}$ on a rotary shaker. The cells were rinsed, plated onto $35-\mathrm{mm}$ dishes (Corning), and expanded to confluence in Eagle's MEM $+10 \%$ fetal bovine serum.

Neuron-oligodendrocyte cultures. Oligodendrocyte cultures were incubated with $0.05 \%$ collagenase (CLSPA grade) in EBSS for $45 \mathrm{~min}$ on a rotary shaker at $35^{\circ} \mathrm{C}$. Any oligodendrocytes that were still adhering were rinsed gently off the dish, the cell suspension was transferred to a tube, and the cells were rinsed with L-15. The cell pellet was resuspended in $0.25 \%$ trypsin in CMFHBSS and incubated for $30 \mathrm{~min}$ on a rotary shaker at $35^{\circ} \mathrm{C}$. Following incubation, trypsin activity was inhibited by adding HIHPS. The cells were rinsed twice in LS medium and then resuspended in Medium 1 at a density of 100,000 oligodendrocytes $/ \mathrm{ml}$. One milliliter of this suspension was plated onto each dorsal root ganglion neuron culture. The purity of this suspension was ascertained by the use of surface immunostaining with mouse monoclonal antibodies to GalC (Raff et al., 1978; Ranscht et al., 1982), in combination with a nuclear dye (Hoescht 33342, Sigma Chemical Co., $10 \mu \mathrm{M}$ ) to distinguish cells from myelin ovoids. Astrocytes were detected by cytoplasmic 
staining with rabbit antibody to GFAP. Approximately $95 \%$ of the cells were found to be GalC + oligodendrocytes, and approximately $2.5 \%$ were GFAP+ astrocytes. Twenty-four hours after replating the oligodendrocytes, the culture medium was changed to a serum-free defined medium (N2; Bottenstein and Sato, 1979) containing $20 \mathrm{~nm}$ triiodothyronine and purified fibroblast growth factor at a dilution empirically determined as optimum for oligodendrocyte proliferation and survival. Fibroblast growth factor was a gift from Burton Wice and was purified from bovine pituitary gland as described previously (Wice et al., 1987). Cultures were maintained on defined medium for 7-14 d before addition of astrocytes. For some experiments, neuron-oligodendrocyte cultures were prepared by adding oligodendrocytes purified by fluorescence-activated cell sorting (as described above) to purified neuronal cultures. The long-term results obtained with such cultures were essentially identical to those obtained when the oligodendrocytes were purified by antimitotic treatment. Initially, however, these cultures were completely devoid of GFAP + astrocytes. After 2 weeks, the medium was changed to MEM containing $10 \%$ fetal bovine serum, $0.4 \%$ glucose, and 50 biological units of crude nerve growth factor (Medium 2).

Neuron-oligodendrocyte-astrocyte cultures. Purified Type 1 astrocytes were replated onto dorsal root ganglion neuron-oligodendrocyte cocultures after the oligodendrocytes had attached to the neurites and had begun to proliferate. For subculturing, astrocytes were removed from the flasks with $0.05 \%$ trypsin in CMFHBSS. The incubation was stopped by addition of soybean trypsin inhibitor plus DNAase, and the cells were washed and resuspended in Dulbecco's Modified Eagle's Medium $+10 \%$ fetal bovine serum. To each neuron-oligodendrocyte culture, 50,000 astrocytes were added in a volume of $1.0 \mathrm{ml}$.

Neuron-oligodendrocyte-fibroblast cultures. Fibroblasts were added to neuron-oligodendrocyte cultures in a manner identical to the astrocyte procedure. The fibroblasts were also added at a density of 50,000 cells per culture.

Preparation of astrocyte-conditioned media. Medium 2 was placed on monolayers of astrocytes (conditioned medium, ACM) or in empty flasks (unconditioned medium, UCM) for $2 \mathrm{~d}$ at $35^{\circ} \mathrm{C}$ in a $5 \% \mathrm{CO}_{2}$ incubator. Media were then filtered through a $0.22-\mu \mathrm{m}$ Millipore filter. For use in experiments, both the ACM and the UCM were supplemented with amino acids, vitamins, fetal bovine serum, glucose, and crude nerve growth factor in amounts equivalent to their original concentrations in Medium 2. The media thus generated contained nutrients at $1 \times$ concentration in Medium 2, $2 \times$ concentration in UCM, and $2 \times-\mathrm{A}$ concentration (where $A$ is the amount of nutrient used by the astrocyte monolayer) present in ACM. In some experiments, ACM was diluted $1: 2(\mathrm{ACM} / 2)$ and $1: 10(\mathrm{ACM} / 10)$ with Medium 2.

Fixation and Sudan black staining. Cultures were fixed 4-6 weeks after the oligodendrocytes had been added to the dorsal root ganglion neurons. Fixation and staining procedures were those used by Wood and Williams (1984). Briefly, cultures to be stained with Sudan black were fixed for $1 \mathrm{hr}$ at room temperature and $24 \mathrm{hr}$ at $4^{\circ} \mathrm{C}$ in $3 \%$ glutaraldehyde in $0.1 \mathrm{M}$ phosphate buffer. Tissue was postfixed in $0.1 \%$ $\mathrm{OsO}_{4}$ in $0.1 \mathrm{M}$ phosphate buffer. Cultures were then rinsed with $0.1 \mathrm{M}$ phosphate buffer, dehydrated, placed in $0.05 \%$ Sudan black in $\mathrm{EtOH}$, and stained for $45 \mathrm{~min}$. Cultures were rehydrated, rinsed, and mounted with glycerin jelly. Myelin was now clearly recognizable in Sudan blackstained preparations with brightfield illumination.

Immunostaining procedures. Cultures were surface stained either with the monoclonal antibody to GalC, used as undiluted hybridoma supernatant, or with the antibody $\mathrm{O}_{1}$ (also specific for GalC; Sommer and Schachner, 1981) provided as an ascites fluid by Dr. Melitta Schachner and used at 1:50 dilution. Following fixation and permeabilization, the cultures were stained to detect astrocytes with rabbit anti-GFAP. The procedure was a modification of the method described by Raff et al. (1978).

Tumor necrosis factor assay. Dr. Junming Le at the New York University Medical Center assayed the ACM for tumor necrosis activity. The assay procedure was previously reported in Feinman et al. (1987). Briefly, An 73 cells obtained from a rhabdomyosarcoma cell line were selected for high sensitivity to tumor necrosis factor. Sensitive cells were exposed directly to undiluted ACM. The ACM contained no cytotoxicity for Ab73 cells.

Data analysis. The presence of myelin in Sudan black-stained cultures was quantified by counting individual myelin segments. Fields of 0.173 $\mathrm{mm}^{2}$ were sampled at $1 \mathrm{~mm}$ apart in both the $\mathrm{x}$ and $\mathrm{y}$ direction, forming a $20 \mathrm{~mm} \times 20 \mathrm{~mm}$ grid. Three-dimensional matrices were created, with the $x$ and $y$ axes representing the position of the field and the $z$ axis representing the quantity of myelin present. Myelin counts over 40 were counted as 40 . Data for cultures grown in similar conditions were averaged for the quantity of myelin present, keeping positional information intact. Three-dimensional histograms were plotted using data averaged from three cultures. Averaging of matrices was performed using Microsoft Excel. Three-dimensional graphing was carried out by Statworks. To transfer data from Excel files to Statworks files, it was necessary to alter the files with a program written in Lightspeed C (graciously written by Alon Mogilner at New York I Jniversity Medical School).

\section{Results}

A prerequisite for these experiments was the preparation of separate cultures of purified neurons, purified oligodendrocytes, purified astrocytes, and fibroblasts as described in Materials and Methods. At the time of addition of the oligodendrocytes, the neuronal cultures contained a central area where the neuronal somata were clustered and from which bare axons had grown radially into the periphery of the culture dish. As visualized in cultures immunostained $24 \mathrm{hr}$ after oligodendrocyte addition, the oligodendrocytes were randomly and sparsely distributed over the neurons and neurite outgrowth. When the oligodendrocytes were purified by the cell-sorting method, GFAP+ astrocytes could not be detected at this time. Immunocytochemical analysis of such cultures 2 weeks after the addition of oligodendrocytes revealed that the oligodendrocytes had increased dramatically in number, blanketing large areas of the culture with their cell bodies and processes (Fig. 1, $a, b$ ). In addition, a few small clusters of stellate-shaped GFAP-positive astrocytes were present. In those cultures receiving oligodendrocytes purified by cell sorting, the astrocytes must have arisen through the proliferation and differentiation of GFAP-negative precursors contaminating the purified oligodendrocyte preparation.

Myelination was first observed in neuron-oligodendrocyte cultures 3 weeks after oligodendrocyte addition and increased in extent over the next 3 weeks. Myelination took the form of clusters of thin, short segments, which were usually found in regions of dense cellularity (e.g., near aggregates of neuronal somata or in large fascicles) and which wcre best visualized after Sudan black staining (Fig. 2, $a, b$ ). Immunocytochemical analysis of parallel cultures at 4 and 6 weeks revealed increased numbers of both oligodendrocytes and astrocytes compared to the 2-week time point, but overall the number of astrocytes remained markedly lower than the number of oligodendrocytes.

To determine the effects of Type 1 astrocytes on myelination, neuron-oligodendrocyte cultures to which Type 1 astrocytes were added were compared to neuron-oligodendrocyte cultures and to neuron-oligodendrocyte-fibroblast cultures. The Type 1 astrocytes or fibroblasts were added 2 weeks after the addition of the oligodendrocytes as described in Materials and Methods. Thus, oligodendrocyte proliferation due to the mitogenic effect of neurons was well underway before the addition of either astrocytes or fibroblasts. Because Type 1 astrocytes and fibroblasts tend to overgrow the cultures in much the same way, the fibroblast-supplemented cultures provided a control for both the nonspecific effects of cellular overloading and the trauma of adding cells to an already established culture. For myelin quantitation, representative cultures from each of these three groups were fixed 4 weeks after the addition of oligodendrocytes and subjected to analysis as described in Materials and Methods.

The results are shown photographically in Figure 2 and graphically in Figure 3. In the cultures to which Type 1 astrocytes were added (Fig. $3 b$ ), there was a marked reduction in the extent 
Figure 1. Oligodendrocytes in a neuron-oligodendrocyte culture 2 weeks after oligodendrocyte addition. $a$, Phase contrast micrograph illustrating that oligodendrocytes appear to interact with the axons in their vicinity. The oligodendrocytes appear as small round phase dark structures aligned along or situated next to neurite processes. $b$, Fluorescence image of the field in $a$ to show immunostaining with monoclonal anti-GalC antibody. Oligodendrocytes extend $\mathrm{GalC}+$ processes to form a complex network in a pattern determined by the axons along which they appear to grow. $\times 500$.
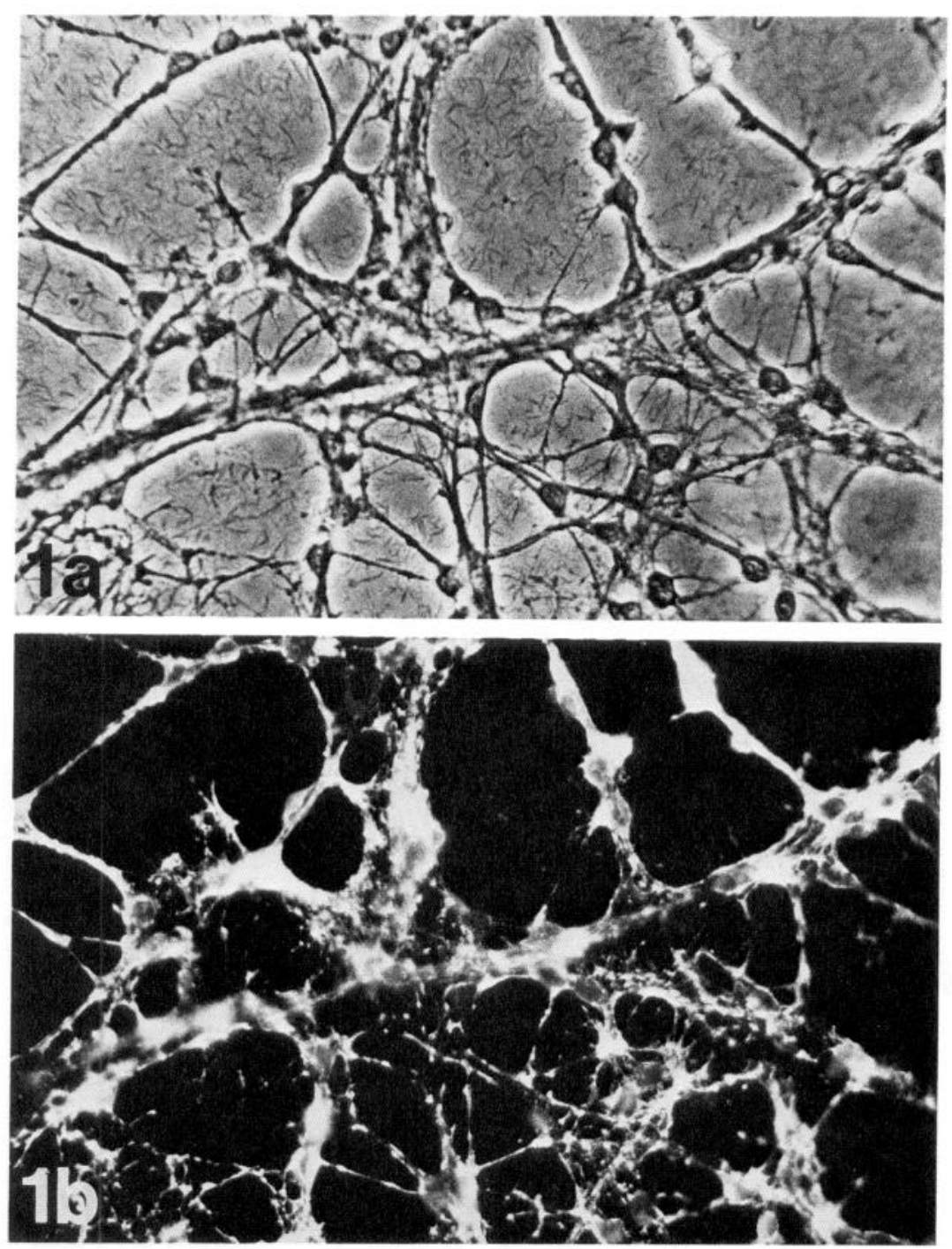

of myelination, compared to that observed in neuron-oligodendrocyte (Fig. $3 a$ ) or neuron-oligodendrocyte-fibroblast cultures (Fig. 3c). It should be noted that the myelin formed in neuron-oligodendrocyte cultures often displayed characteristic irregularities, including membranous extrusions (Fig. $2 b$ ). In contrast, the myelin in neuron-fibroblast cultures was more regular (Fig. $2 e, f$ ). The ability of the oligodendrocytes to function in the presence of large numbers of fibroblasts demonstrated that the inhibition by Type 1 astrocytes was a cell-specific interaction. Immunostaining revealed that the neuron-oligodendrocyte-Type 1 astrocyte cultures were covered by an almost confluent layer of GFAP+ cells, many of which were polygonal rather than stellate (Fig. 4, $a, b$ ). This was in contrast to the loose meshwork and scattered islands of stellate GFAP+ cells observed in controls (Fig. 4, $c, d$ ).

Oligodendrocyte numbers were not quantitated in these experiments but appeared to be markedly reduced in neuronoligodendrocyte-Type 1 astrocyte cultures compared to controls. The number of oligodendrocytes present in neuron-oligodendrocyte-fibroblast cultures did not appear different from that in neuron-oligodendrocyte cultures. A precise comparison of the number of oligodendrocytes present in myelinated cul- tures was not made because penetration of the antibody used for immunostaining was blocked by the presence of either astrocytes or fibroblasts and because the growth of long, branching oligodendrocyte processes over neighboring cells made it impossible to discern which cells were $\mathrm{GalC}+$, especially in areas of high cell density. In a single experiment, we attempted to quantitate oligodendrocyte cultures stained 1 week after the addition of astrocytes. The data from this experiment was intriguing. The number of oligodendrocytes in isolation from other oligodendrocytes or in small clusters (up to 10 cells) was similar in neuron-oligodendrocyte and neuron-oligodendrocyte-Type 1 astrocyte cultures (average $48 / \mathrm{mm}^{2}$ in neuron-oligodendrocyte, $50 / \mathrm{mm}^{2}$ in neuron-oligodendrocyte-Type 1 astrocyte cultures), but large colonies of oligodendrocytes (with more than 20 oligodendrocytes/high magnification field) were 6-fold more numerous in neuron-oligodendrocyte cultures than in neuron-oligodendrocyte-Type 1 astrocyte cultures $\left(1.9 / \mathrm{mm}^{2}\right.$ in neuron-oligodendrocyte vs $0.3 / \mathrm{mm}^{2}$ in neuron-oligodendrocyte-Type 1 astrocyte cultures). The coexistence of many isolated or small clusters of oligodendrocytes, which survive but apparently do not divide, with large colonies of dividing oligodendrocytes in a neuron-oligodendrocyte culture is a normal 

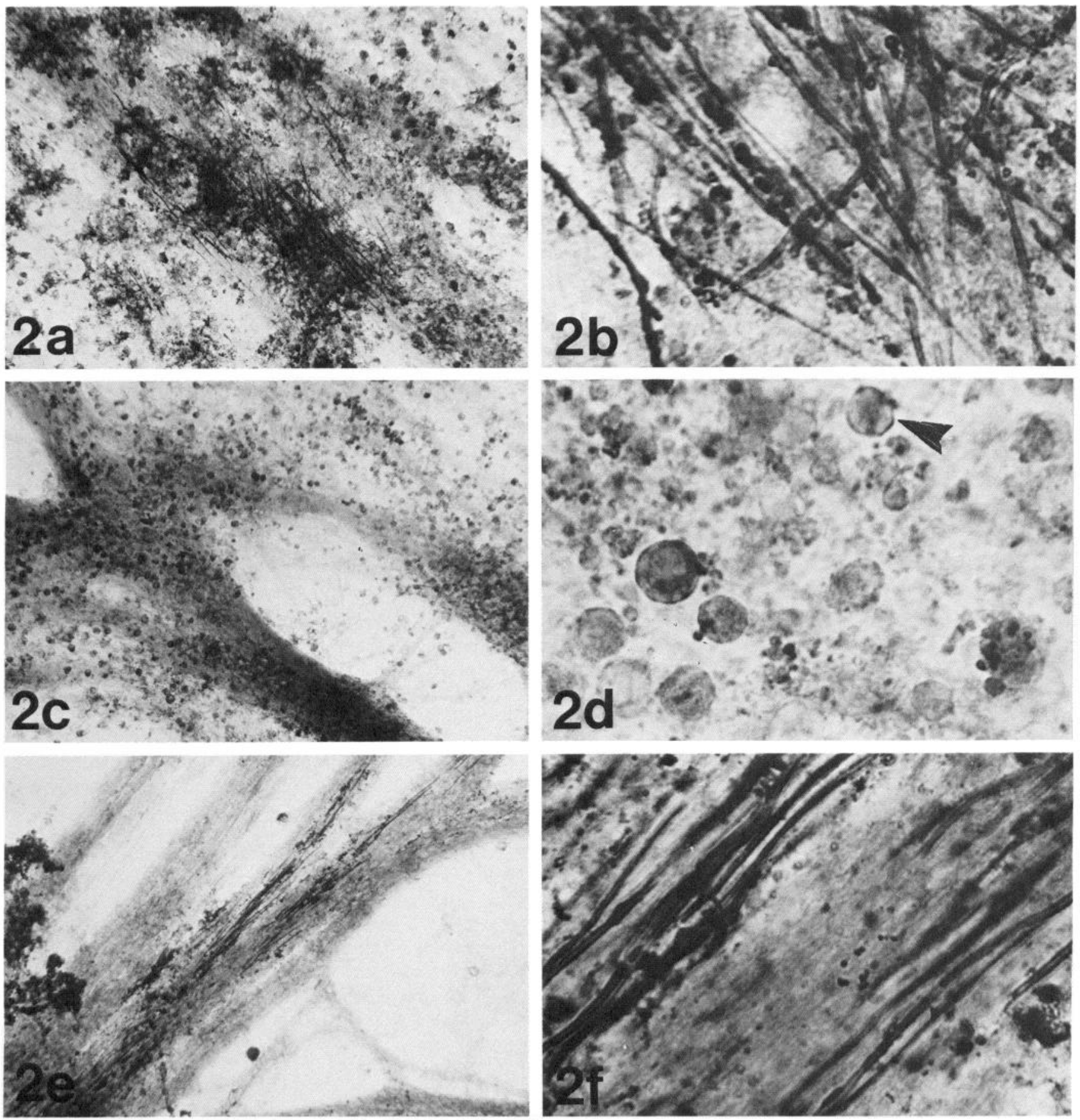

Figure 2. Myelination in neuron-oligodendrocyte, neuron-oligodendrocyte-Type 1 astrocyte, and neuron-oligodendrocyte-fibroblast cultures. $a$, Low and, $b$, high magnification of an area of myelination in a neuron-oligodendrocyte culture 6 weeks after the addition of oligodendrocytes. Myelin segments were generally found in areas of high cell density and were short, thin, and irregular. $c$, Low and, $d$, high magnification of an area in a neuron-oligodendrocyte-Type 1 astrocyte culture comparable in cellular organization and density to that shown in $a$ and $b$. Myelin sheaths were absent despite the presence of cells that looked like oligodendrocytes (arrow) and many axons. $e$, Low and, $f$, high magnification micrographs of a comparable area in a neuron-oligodendrocyte-fibroblast culture, showing that the overgrowth of a third cell type does not, as a culture artifact, inhibit myelination. Sudan black stained. $a, c, e \times 120 . b, d, f \times 890$.

feature of this type of culture and has been previously described (Wood and Bunge, 1986b). The present result might indicate that it is the continued division of oligodendrocytes within these colonies that is blocked by the astrocytes, whereas the survival of individual oligodendrocytes is not affected.
The effects of the physical presence of Type 1 astrocytes on oligodendrocyte function could be reproduced by maintaining neuron-oligodendrocyte cultures on Type 1 ACM. Myelination was markedly inhibited by ACM in a dose-dependent manner (Fig. 5), and the number and morphological appearance of the 

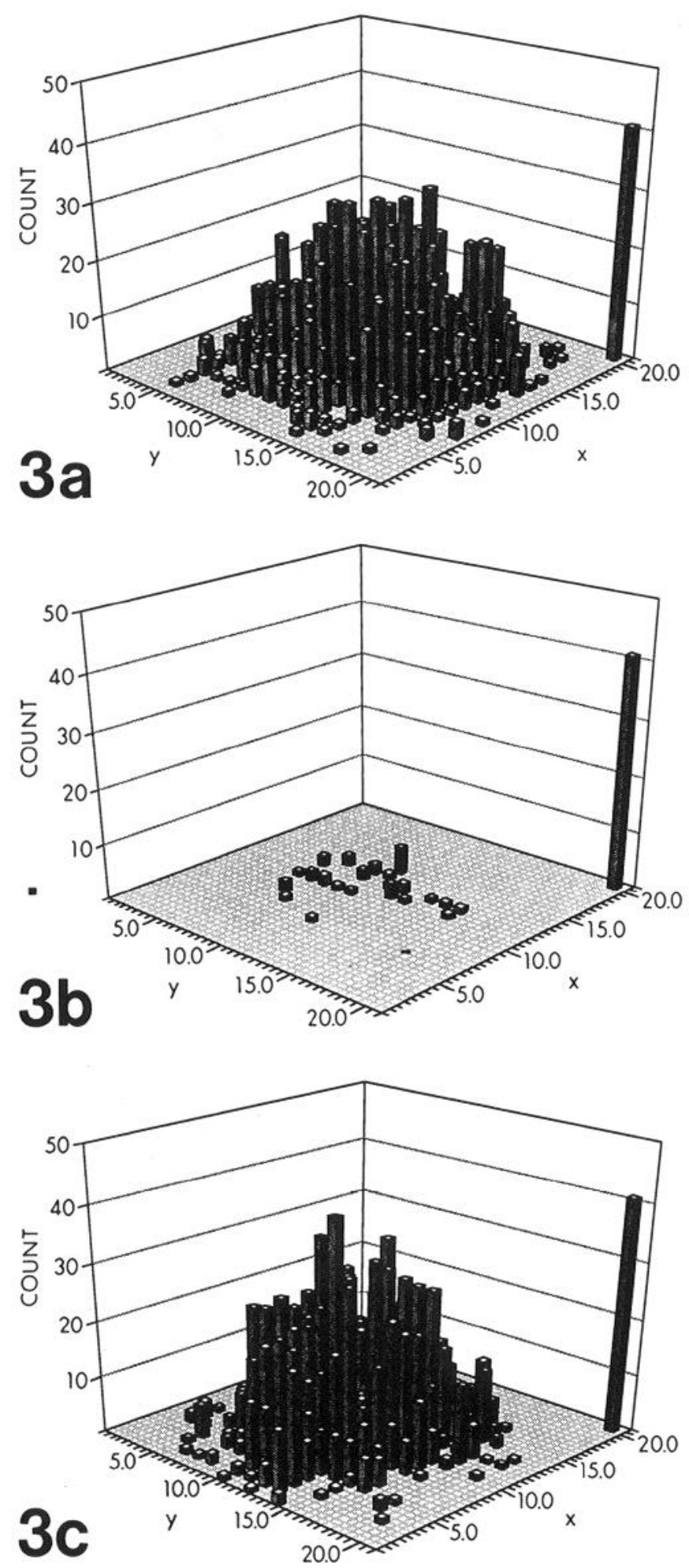

Figure 3. Three-dimensional representation of the amount of myelin present in cultures with $(a)$ neurons and oligodendrocytes (total number of myelin segments counted was $1968 \pm 482)$, (b) neurons, oligodendrocytes, and astrocytes (total number of myelin segments counted was $40 \pm 36$ ), and $(c)$ neurons, oligodendrocytes, and fibroblasts (total number of myelin segments counted was $2050 \pm 617$ ). The $x$ and $y$ axes represent the plane of the culture. The $\mathrm{z}$ axis represents the average quantity of myelin that was present in 3 cultures at the given $x$ and $y$ coordinate. A total of 400 fields/culture was counted. These 3-d histograms demonstrate the large amounts of myelin that typically form in the neuron-oligodendrocyte cultures $(a)$. The addition of astrocytes oligodendrocytes in neuron-oligodendrocyte cultures fed undiluted ACM were similar to those to which Type 1 astrocytes had been added. These results demonstrate that the effects of the Type 1 astrocytes were mediated by a factor released into the culture medium. Preliminary experiments to further characterize the factor(s) have provided evidence that the factor responsible for inhibiting myelination is heat stable (i.e., it retained activity after $10 \mathrm{~min}$ at $100^{\circ} \mathrm{C}$ ) but nondialyzable. Interestingly, in the presence of dialyzed ACM, oligodendrocyte proliferation did not appear to be suppressed, suggesting that the effects of ACM on myelination and proliferation may be due to different factors. In view of recently reported evidence (Robbins et al., 1987) that astrocytes can secrete a factor with tumor necrosis factor activity that is specifically lethal to oligodendrocytes, ACM was assayed for this activity. No detectable tumor necrosis activity was produced by the Type 1 astrocytes under the conditions used to generate the conditioned medium used in our experiments.

\section{Discussion}

The data presented here demonstrate that soluble factors secreted by Type 1 astrocytes can, in some situations, inhibit the production of myelin sheaths by oligodendrocytes and may, in addition, interfere with oligodendrocyte proliferation. The Type 1 astrocytes used in these experiments were prepared, as nearly as possible, by the same method used by others to prepare the astrocytes in experiments that demonstrated the secretion by those astrocytes of a factor (platelet-derived growth factor) that promoted the division of oligodendrocyte progenitors (Noble et al., 1988; Raff et al., 1988; Richardson et al., 1988). At the present time, we cannot provide a proven explanation of this apparent discrepancy, but we would emphasize a major difference between the present experiments and those demonstrating supportive influences. The oligodendrocytes used here were obtained from adult animals, whereas the progenitors were obtained from newborn animals. Other work from this laboratory with oligodendrocytes from adult animals has shown that most of the new oligodendrocytes generated in vitro were derived from a subpopulation of these mature oligodendrocytes and not from progenitors (Wood and Bunge, 1986b). It is possible that astrocytes growing in culture dishes away from appropriate regulatory influences may remain in a reactive state, caused by the physical disruption of the tissue. These "reactive" astrocytes may release a variety of factors, including some with potentially inhibitory or even toxic effects on mature oligodendrocytes. The slight inhibition of myelination observed even with 10 -fold diluted $\mathrm{ACM}$ argues that the differing results did not derive from gross differences in the concentration of a single factor (if, for example, a single factor was stimulatory at low concentrations and inhibitory at higher concentrations). It might also be worthwhile to consider whether oligodendrocytes might become, with maturation, sensitized to some factor released by the astrocytes that was innocuous for immature oligodendrocytes.

It is important to consider the different uses and possible effects of serum in the experiments reported here and in the progenitor experiments. We have consistently observed that se-

\section{$\leftarrow$}

(b) but not fibroblasts (c) inhibits myelin formation. (The 40-unit-high column at position 20,20 standardizes the $\mathrm{z}$ axis and does not represent any data.) 

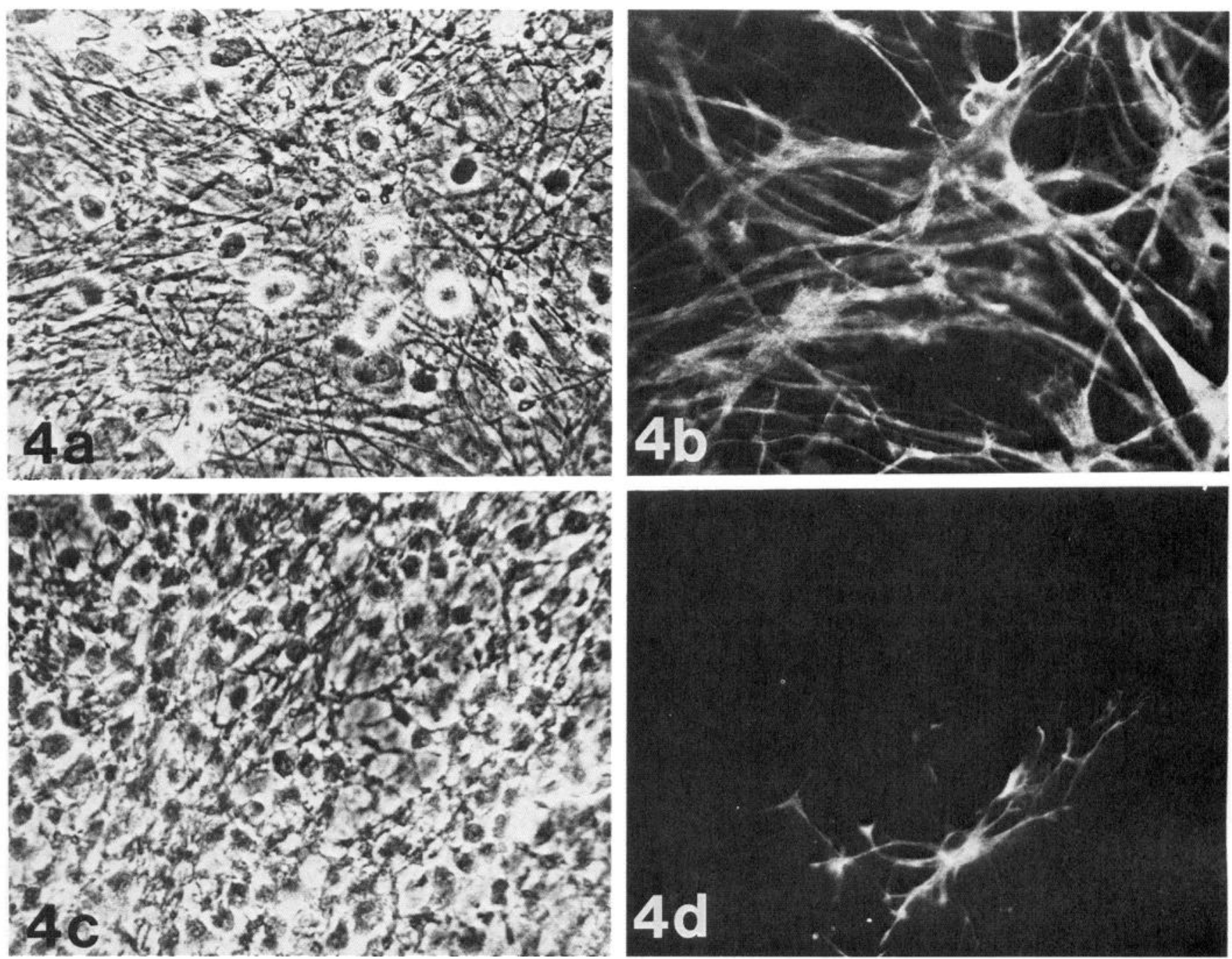

Figure 4. Comparison of the astrocyte populations in neuron-oligodendrocyte and neuron-oligodendrocyte-Type 1 astrocyte cultures. $a$, Phase and, $b$, fluorescence micrographs of a neuron-oligodendrocyte-Type 1 astrocyte culture 6 weeks after the addition of oligodendrocytes. Astrocytes were undetectable by phase microscopy $(a)$ against the background of neurites and phase dark oligodendrocytes. By immunofluorescence $(b)$, a confluent layer of large, often polygonal astrocytes was visible. In neuron-oligodendrocyte cultures ( $c$, phase contrast; $d$, fluorescence), the few astrocytes that contaminated the neurons-oligodendrocytes were usually stellate in appearance. The cultures were immunostained with anti-GFAP. $\times 500$.

rum is required for optimal long-term differentiation of oligodendrocytes, possibly by enhancing neuronal survival and maturation, and that the presence of serum does not appear to block the proliferation of oligodendrocytes from mature animals. On the other hand, the development of oligodendrocytes from progenitors is apparently blocked by serum, which causes the conversion of progenitors into Type 2 astrocytes. The effect of serum on the progenitors has never been fully explained. The ACM that was shown to contain PDGF and to stimulate progenitor division was thus, by necessity, either serum-free or low in serum (Noble et al., 1988; Richardson et al., 1988). Furthermore, the observation of factors that inhibited astrocyte proliferation in serum-containing but not in serum-free medium from cultures that contained predominantly astrocytes has been reported previously (Aloisi et al., 1987). Paradoxically, astrocytes are generally observed to proliferate better in serum-containing than in serum-free medium. These observations suggest that the nature of the molecules released by astrocytes might be governed by parameters, such as the length of time in culture and the cell density, as well as the medium composition.

From these and other considerations, it is apparent that much additional work is needed if we are to understand the full potential of astrocytes, especially as they may affect the functioning of other cells in their vicinity. In our own earlier work, for example, we used neuronal cultures of the same type employed in the present experiments and added mixed glial populations derived from embryonic spinal cord tissue. In these experiments, astrocyte and oligodendrocyte development occurs in parallel, and the mature cultures contain substantial numbers of astrocytes in close association with myelinated axonal fascicles, yet no inhibition of oligodendrocyte function was observed (Wood and Williams, 1984).

Since neurons and neurites are intensely positive for the antigen (A2B5) by which Type 2 astrocytes are identified (Raff et al., 1983), we did not attempt to determine the phenotypes of the astrocytes in "neuron-oligodendrocyte" cultures. In unpub- 

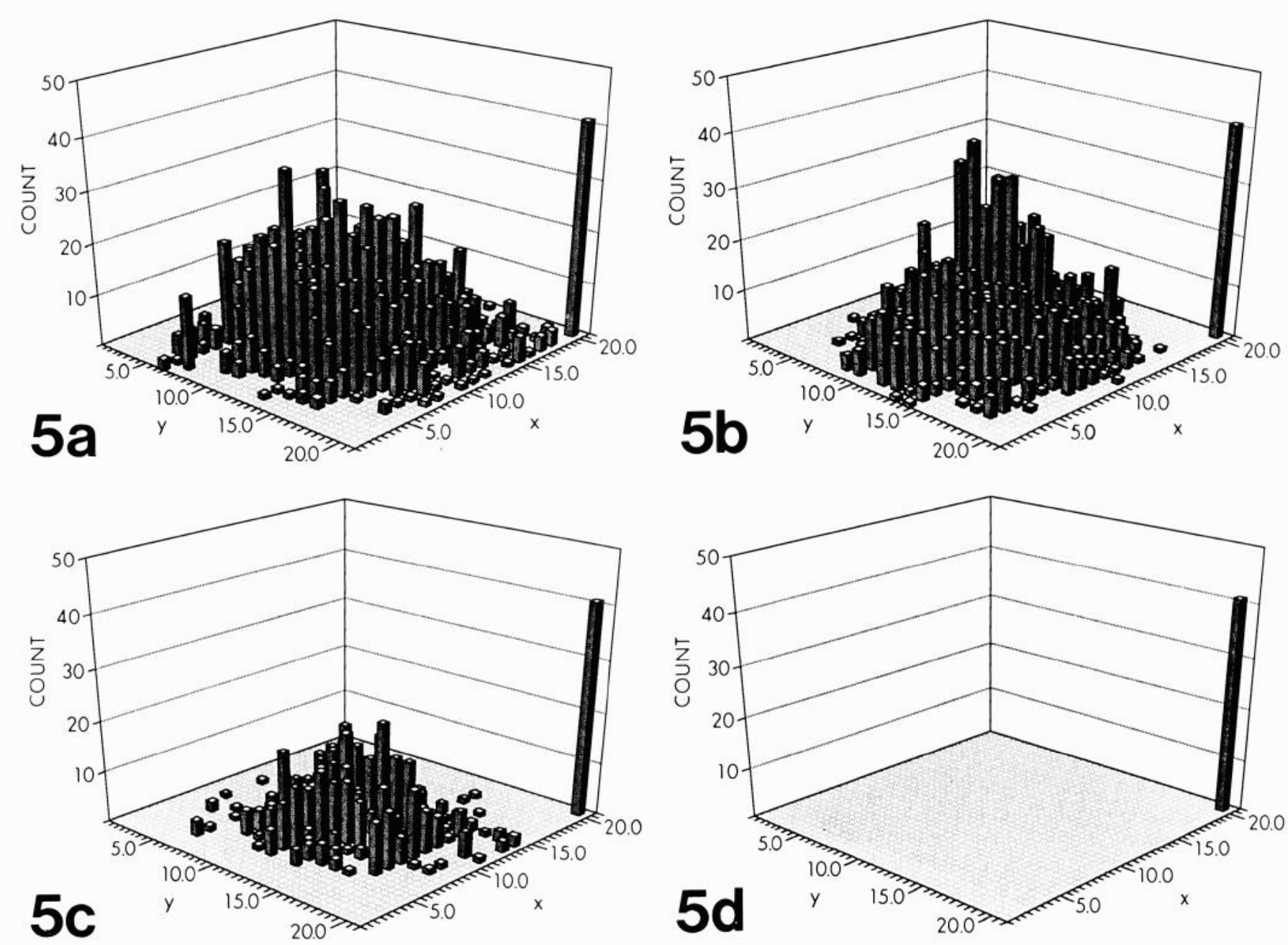

Figure 5. Dose-dependent inhibition of myelin formation by astrocyte-conditioned medium. $a$, Control neuron-oligodendrocyte culture (total number of myelin segments counted was $2071 \pm 998$ ). $b$. Astrocyte-conditioned medium diluted 1:10 (total number of myelin segments counted was $1500 \pm 200$ ). $c$, Astrocyte-conditioned medium diluted $1: 2$ (total number of myelin segments counted was $636 \pm 928$ ). $d$, Astrocyte-conditioned medium undiluted (total number of myelin segments counted was $0 \pm 0$ ). Myelin quantitation and graphics are as described in Figure 3 .

lished experiments separate from those reported here, when the purified adult oligodendrocyte preparation was plated and maintained on a collagen substrate without neurons, the astrocyte population that developed was composed of approximately equal numbers of Types 1 and 2 astrocytes. This suggests that neuronoligodendrocyte culture probably contained some Type 2 astrocytes as well. However, the extensive myelination observed in "neuron-oligodendrocyte" cultures indicates that the relatively few astrocytes that grew as contaminants of the cultures had negligible or only minor effects on oligodendrocyte function. When there was a larger indigenous contamination by astrocytes, the astrocytes were usually located in the peripheral parts of the culture, and these areas often lacked myelin segments. Recently, a procedure for obtaining cultures enriched in Type 2 astrocytes has been reported (Aloisi et al., 1988). It would now be of considerable interest to determine if Type 2 astrocytes also are capable of releasing inhibitory factors under conditions similar to those used here. Our data strongly suggest that Type 1 astrocytes can, in some instances, interfere with the production of myelin by oligodendrocytes. Perhaps this is a partial expla- nation for the limited amount of remyelination that occurs in plaques associated with multiple sclerosis.

Several important and still unanswered questions are raised by our results. Most notably, how would large numbers of Type 2 astrocytes affect oligodendrocyte myelination? Would oligodendrocytes obtained from optic nerve or from a younger animal respond differently in this situation? Can the putative factor(s) secreted by astrocytes be isolated and characterized further? Are these factor(s) present in demyelinated regions of the CNS? The answers to these questions should significantly improve our understanding of the contribution of astrocytes to the pathology of and potential for recovery from disease or injury in the CNS.

\section{References}

Aloisi, F., C. Agresti, and G. Levi (1987) Glial conditioned media inhibit the proliferation of cultured rat cerebellar astrocytes. Neurochem. Res. 12: 189-195.

Aloisi, F., C. Agresti, and G. Levi (1988) Establishment, characterization, and evolution of cultures enriched in type 2 astrocytes. J. Neurosci. Res. 21: 188-198.

Bignami, A., L. F. Eng, D. Dahl, and C. T. Uyeda (1972) Localization 
of the glial fibrillary acidic protein in astrocytes by immunofluorescence. Brain Res. 43: 429-435.

Blakemore, W. F. (1978) Observations on remyelination in the rabbit spinal cord following demyelination induced by lysolecithin. Neuropathol. Appl. Neurobiol. 4: 47-59.

Bornstein, M. B. (1958) Reconstituted rat-tail collagen used as a substrate for tissue cultures on coverslips. Lab. Invest. 7: 134-137.

Bottenstein, J. E., and G. H. Sato (1979) Growth of a rat neuroblastoma cell line in serum-free supplemented medium. Proc. Natl. Acad. Sci. USA 76: 514-517.

Feinman, R., D. Henriksen-DeStefano, M. Tsujmoto, and J. Vilcek (1987) Tumor necrosis factor is an important mediator of tumor cell killing by human monocytes. J. Immunol. 138: 635-640.

ffrench-Constant, C., and M. Raff (1986) The oligodendrocyte-type 2 astrocyte cell lineage is specialized for myelination. Nature 323: $325-$ 328.

Ludwin, S. K. (1981) Pathology of demyelination and remyelination. In Demyelinating Disease: Basic and Clinical Electrophysiology, S G. Waxman and S. M. Ritchie, eds., pp. 123-187, Raven, New York.

McCarthy, K., and J. de Vellis (1980) Preparation of separate astroglial and oligodendroglial cell cultures from rat cerebral tissue. J. Cell. Biol. 85: 890-902.

Miller, R. H., S. David, R. Patel, E. Abney, and M. C. Raff (1985) A quantitative immunohistochemical study of macroglial cell development in rat optic nerve: in vivo evidence for two distant astrocyte lineages. Dev. Biol. 111: 35-41.

Miller, R. H., E. R. Abney, S. David, C. F. Constant, R. Lindsay, R. Patel, J. Stone, and M. C. Raff (1986) Is reactive gliosis a property of a distinct subpopulation of astrocytes? J. Neurosci. 6: 22-29.

Noble, M., J. Fok-Seang, and J. Cohen (1984) Glia are a unique substrate for the in vitro growth of central nervous system neurons. J. Neurosci. 4: 1892-1903.

Noble, M., K. Murray, P. Stroobant, M. Waterfield, and F. Riddle (1988) Platelet-derived growth factor promotes division and motility and inhibits premature differentiation of the oligodendrocyte/type 2 astrocyte progenitor cell. Nature 333: 560-562.

Prineas, J. (1985) The neuropathology of multiple sclerosis. In Handbook of Clinical Neurology, Vol. 3, Demyelinating Diseases, J. C. Koestler, ed., pp. 213-257, Elsevier, Amsterdam.

Raff, M. C., R. Mirsky, and K. L. Fields (1978) Galactocerebroside is a specific cell-surface antigenic marker for oligodendrocytes in culture. Nature 274: 813-816.

Raff, M. C., K. L. Fields, S.-I. Hakomori, R. Mirsky, R. M. Pruss, and
J. Winter (1979) Cell type-specific markers for distinguishing and studying neurons and the major classes of glial cells in culture. Brain Res. 174: 283-308.

Raff, M. C., E. R. Abney, J. Cohen, R. Lindsay, and M. Noble (1983) Two types of astrocytes in cultures of developing white matter: Differences in morphology, surface gangliosides, and growth characteristics. J. Neurosci. 3: 1289-1300.

Raff, M. C., E. R. Abney, and R. H. Miller (1984) Two glial cell lineages diverge prenatally in rat optic nerve. Dev. Biol. 106: 53-60.

Raff, M., L. Lillien, W. Richardson, J. Burne, and M. Noble (1988) Platelet-derived growth factor from astrocytes drives the clock that times oligodendrocyte development in culture. Nature 333:562-565.

Ranscht, B., P. A. Clapshaw, J. Price, M. Noble, and W. Seifert (1982) Development of oligodendrocytes and Schwann cells studied with a monoclonal antibody against galactocerebroside. Proc. Natl. Acad. Sci. USA 79: 2709-2713.

Richardson, W. D., W. Pringle, M. J. Mosley, B. Westermark, and M. Dubois-Dalcq (1988) A role for platelet-derived growth factor in normal gliogenesis in the central nervous system. Cell 53: 309-319.

Robbins, D. S., Y. Shirazi, B. Drysdale, A. Lieberman, H. S. Shin, and M. L. Shin (1987) Production of cytotoxic factor for oligodendrocytes by stimulated astrocytes. J. Immunol. 139: 2593-2597.

Skoff, R., D. Price, and A. Stocks (1976) Electron microscopic autoradiographic studies of gliogenesis in rat optic nerve. II. Time of origin. J. Comp. Neurol. 169: 313-333.

Sommer, I., and M. Schachner (1981) Monoclonal antibodies (01-04) to oligodendrocyte cell surfaces. An immunocytological study in the central nervous system. Dev. Biol. 83: 311-327.

Wice, B., J. Milbrandt, and L. Glaser. (1987) Control of muscle differentiation in $\mathrm{BC} 3 \mathrm{Hl}$ cells by fibroblast growth factor and Vanodate. J. Biol. Chem. 262: 1810-1817.

Wood, P. M. (1976) Separation of functional Schwann cells and neurons from normal peripheral nerve tissue. Brain Res. 115: 361-375.

Wood, P., and R. P. Bunge (1986a) Evidence that axons are mitogenic for oligodendrocytes isolated from adult animals. Nature 320: 756758.

Wood, P. M., and R. P. Bunge (1986b) Myelination of cultured dorsal root ganglion neurons by oligodendrocytes obtained from adult rats. J. Neurol. Sci. 74: 153-169.

Wood, P. M., and A. K. Williams (1984) Oligodendrocyte proliferation and CNS myelination in cultures containing dissociated embryonic neuroglia and dorsal root ganglion neurons. Dev. Brain Res. 12: 225241. 